\title{
Le Sommet national sur une politique en matière d'information : bilan et perspectives
}

\section{The National Summit on Information Policy: Summary and} Perspectives

\section{La conferencia nacional sobre una política en materia de información: panorama y perspectivas}

\section{Claude Bonnelly et Bernard Dumouchel}

Volume 39, numéro 3, juillet-septembre 1993

URI : https://id.erudit.org/iderudit/1028750ar

DOI : https://doi.org/10.7202/1028750ar

Aller au sommaire du numéro

Éditeur(s)

Association pour l'avancement des sciences et des techniques de la documentation (ASTED)

ISSN

0315-2340 (imprimé)

2291-8949 (numérique)

Découvrir la revue

Citer cet article

Bonnelly, C. \& Dumouchel, B. (1993). Le Sommet national sur une politique en matière d'information : bilan et perspectives. Documentation et bibliothèques, 39(3), 153-159. https://doi.org/10.7202/1028750ar
Résumé de l'article

Le Sommet national sur une politique en matière d'information, tenu à Ottawa du 6 au 8 décembre 1992, était une première au Canada. Cette rencontre qui réunissait des représentants de tous les milieux intéressés n'a pas produit, sur le champ, une politique en matière d'information, cependant elle en a posé des jalons importants. Les auteurs, membres du groupe chargé de définir le cadre et les objectifs de ce sommet, dressent un bilan de l'événement et dégagent quelques perspectives après en avoir relaté la genèse. En complément, suit la liste des recommandations extraites du Résumé du Sommet national sur une politique en matière d'information, 1992 paru en mai 1993.
Tous droits réservés (c) Association pour l'avancement des sciences et des techniques de la documentation (ASTED), 1993
Ce document est protégé par la loi sur le droit d'auteur. L'utilisation des services d’Érudit (y compris la reproduction) est assujettie à sa politique d'utilisation que vous pouvez consulter en ligne. 


\title{
Le Sommet national sur une politique en matière d'information : bilan et perspectives
}

\author{
Claude Bonnelly \\ Bibliothèque de l'Université Laval \\ Bernard Dumouchel \\ Institut canadien de l'information scientifique et technique
}

Le Sommet national sur une politique en matière d'information, tenu à Ottawa du 6 au 8 décembre 1992, était une première au Canada. Cette rencontre qui réunissait des représentants de tous les milieux intéressés n'a pas produit, sur le champ, une politique en matière d'information, cependant elle en a posé des jalons importants. Les auteurs, membres du groupe chargé de définir le cadre et les objectifs de ce sommet, dressent un bilan de l'événement et dégagent quelques perspectives après en avoir relaté la genèse. En complément, suit la liste des recommandations extraites du Résumé du Sommet national sur une politique en matière d'information, 1992 paru en mai 1993.

\section{The National Summit on Information Policy : Summary and Perspectives}

The National Summit on Information Policy, held in Ottawa (December 6-8, 1992), was a first for Canada. The conference brought together interested parties and, while it did not lead to the instantaneous creation of an information policy, it did help establish the principle tenants. The authors, members of a group responsable for defining the framework and the objectives of the summit, present its history, summarise the event and outline a few perspectives. The recommendations from the Summary Report of the National Summit on Information Policy,1992 published in May 1993, are included.
La conferencia nacional sobre una política en materia de información : panorama y perspectivas.

La primera conferencia nacional sobre la política en materia de información tuvo lugar en Ottawa del 6 al 8 de diciembre de 1992. Este encuentro que reunía a representantes de todos los medios interesados no ha producido inmediatamente una política en materia de información, pero ha preparado el terreno de manera muy apreciable. Los autores, miembros del grupo responsable para definir los objetivos de esta conferencia, hacen un resumen de este encuentro, incluyendo la génesis y algunas perspectivas. A continuación, sigue la lista de las recomendaciones tomadas del Résumé du Sommet national sur une politique en matière d'information, 1992, publicado en mayo de 1993.
Du 6 au 8 décembre 1992 s'est tenu à Ottawa le Sommet national sur une politique en matière d'information. Événement majeur, première initiative de ce genre au Canada, le Sommet a réuni quelque 300 personnes, délégués et observateurs, qui ont discuté des enjeux et des défis majeurs en matière d'information au Canada. Ces délégués représentaient des organismes de divers milieux : bibliothèques, industries culturelles, éducation, technologie de l'information et des communications, services d'information, édition, groupes de consommateurs, etc. Après quelques mois seulement, il serait sans doute prématuré et plutôt présomptueux d'évaluer les retombées de ce Sommet; il nous apparaît utile cependant, sans reprendre ce qui a déjà été écrit sur le sujet, de dresser un bref bilan de l'entre- prise et de dégager quelques perspectives.

II est d'abord opportun de rappeler que ce Sommet a été le fruit d'une longue gestation qui, par les réflexions et discussions souvent animées quil'ont accompagnée, a certes contribué à préparer les mentalités et à donner aux délibérations de décembre 1992 à Ottawa un caractère si positif.

\section{Historique}

C'est en 1986, lors du congrès conjoint ASTED/CLA tenu à Québec, que fut formulée pour la première fois la proposition d'organiser un Sommet sur les bibliothèques et les services d'information documentaire. On s'appuyait alors sur le modèle de la White House Conference on Library and Information Services, qui avait eu lieu aux États-Unis en 1979, et qui fut d'ailleurs renouvelée en 1991. On allait aussi par la suite s'inspirer de la fameuse Glenerin Declaration, établie par des experts canadiens, américains et britanniques, qui affirmaient notamment :

We have moved from an industrial to an information age, where the efficient exploitation of information as an economic resource and a sector of production has become crucial to the achievement of economic growth ... the production, distribution and use of information have become matters of strategic economic, social and political importance. To ensure that the benefits of the information age are fully realized, 
it is necessary to create and maintain an environment which provides for the open and unrestricted exchange of information ${ }^{1}$.

Cette déclaration définissait d'emblée le contexte ainsi que l'objectif général qui devaient orienter toutes les discussions ultérieures.

Dès 1987, un comité consultatif fut formé en vue d'examiner la faisabilité d'un tel Sommet. Ce comité était composé de représentants de la CLA, de l'ASTED, de l'Association des bibliothèques de recherche du Canada, de l'Association canadienne des sciences de l'information, de la Canadian Association of College and University Libraries, de la Canadian Association of Special Libraries and Information Services, du Council of Administration of Large Urban Public Libraries, du Provincial/Territorial Library Directors Council, de l'Ontario Library Association, de la Special Libraries Association, de la Canadien Library Trustee's Association, de la Bibliothèque nationale du Canada et del'Institut canadien de l'information scientifique et technique (ICIST). Grâce à des subventions obtenues de la Bibliothèque nationale et de I'ICIST, une firme de consultants, Enterprise Canada, fut retenue en vue d'élaborer une proposition concrète. Enterprise Canada déposa en avril 1988 son rapport intitulé «A Proposal for a national Summit Conference on Libraries and Information Services" ${ }^{2}$ et proposa que l'événement soit tenu en 1990. Ce document, transmis aux associations et organismes concernés, a suscité plusieurs débats et n'a pas recueilli l'unanimité. On s'interrogeait sur l'orientation générale du projet, on avait du mal à saisir la portée réelle des objectifs, on s'inquiétait des modes d'organisation et de la faisabilité, bref, l'organisation d'un tel sommet était nettement perçue comme prématurée. Constatant le peu d'appui accordé au projet tel que soumis, le comité consultatif a convenu de ne pas donner suite à la proposition d'Enterprise Canada mais de poursuivre plutôt la réflexion en confiant à un groupe restreint le soin de dégager une meilleure vue d'ensemble du sommet et d'en préciser les objectifs. $\mathrm{Ce}$ groupe était composé de Jane Cooney, représentante de la CLA, Gwynneth Evans, représentante de la Bibliothèque nationale, et des deux auteurs de cet article,
Claude Bonnelly et Bernard Dumouchel, respectivement représentants de l'ASTED et de I'ICIST. Au terme de travaux intensifs, ce groupe déposa à son tour au printemps 1989 un rapport intitulé: Les partenaires de l'information; sommet national sur les services dinformation au Canada $^{3}$. Comme l'indique sans équivoque le titre du document, ce rapport proposait, sinon un nouveau concept, du moins une vision éclatée et un cadre élargi. Alors qu'auparavant le focus était plutôt centré sur le rôle des bibliothèques dans la société de l'information, l'accent était mis désormais sur la nature même de l'information dans la société de demain et sur les rapports de complémentarité entre les divers intervenants. Le but visé était d'offrir une tribune où tous les intervenants du monde de l'information (producteurs, fournisseurs, diffuseurs, entreprises de services et bibliothèques, gouvernements et utilisateurs) pourraient échanger leurs vues sur le rôle de l'information dans la société contemporaine, clarifier leur part de responsabilité en matière de production, de diffusion et d'accès, discuter des nouveaux défis, définir des orientations et mettre au point des stratégies pour faire face adéquatement aux enjeux des prochaines décennies, dans l'intérêt du public et dans le respect de la complémentarité de tous. Le document définissait en ce sens des objectifs précis, décrivait les résultats escomptés et identifiait les partenaires et auditoires-cibles. II n'est pas inutile de rappeler ici les objectifs généraux tels que définis par le groupe.

Offrir une tribune où les producteurs, les fournisseurs, les représentants des secteurs de soutien et les utilisateurs:

1- puissent échanger leurs vues sur le rôle de l'information dans la société canadienne;

2- puissent clarifierleurs rôles, et discuterles thèmes et les défis qui les préoccupent mutuellement;

3- puissent mettre au point des stratégies qui permettront de faire face aux enjeux, tout en proposant et en orientant les projets et les développements à venir ${ }^{4}$

Ce concept élargi allait désormais offrir de nouvelles balises au projet.
Ce rapport fut transmis aux associations nationales et provinciales du domaine de la bibliothéconomie et des sciences de l'information, de même qu'à la Bibliothèque nationale du Canada et à I'ICIST, qui étaient invitées à formuler leurs réactions. Le groupe visait à obtenir l'assentiment du milieu des bibliothèques, somme toute initiateur du projet, avant d'amorcer les consultations auprès des autres partenaires identifiés. Les réponses furent nombreuses mais mitigées. Bien qu'on approuvait de façon générale l'orientation proposée, certains craignaient l'élargissement du cadre, d'autres s'interrogeaient sur la complexité de l'organisation, certains doutaient de l'efficacité d'un tel forum d'échanges et de discussions; peu d'intervenants se montraient disposés à s'engager concrètement dans la réalisation du projet. En somme, faute d'un consensus général, l'organisation d'un Sommet paraissait encore prématuré. L'élan intégrateur susceptible de rassembler les énergies nécessaires faisait étrangement défaut. L'idéetoutefois poursuivait son chemin. En octobre 1989, dans un ultime effort pour rassembler les volontés, l'administratrice de la Bibliothèque nationale Marianne Scott, de concert avec I'ICIST, réunit à Ottawa un certain nombre de personnes dans le but de prendre une décision finale dans le sens de la réalisation du Sommet ou encore de l'abandon du

1. Barry Lesser et Louis Vagianos, The Glenerin Report : Access, Information Distribution, Efficiency and Protection, Halifax, Institute for Research on Public Policy, 1987, p.4

2. Enterprise Canada, A Proposal for a National Summit Conference on Libraries and Information Services, Ottawa, Canada, June 1990, 1988. $12 \mathrm{p}$.

3. Les partenaires de l'information: Sommet national sur les services d'information au $\mathrm{Ca}$ nada; Partners in information: National Summit on Information Services for Canadians, par/by Claude Bonnelly, (ASTED), Jane Cooney(CLA), Bernard Dumouchel (ICIST) et Gwynneth Evans (BNC), Mai 1989, 12 p.

4. Ibid., p. 2 
projet $^{5}$. Cette réunion d'une journée fut déterminante. Elle a permis de dégager un consensus sur la pertinence de la tenue du Sommet mais aussi de préciser qu'un tel événement n'aurait de réel impact que s'il visait à influencer les stratégies pour une politique en matière d'information. Cette réunion donna donc au projet un éclairage beaucoup plus ciblé et apporta surtout l'élan et le dynamisme nécessaires à sa poursuite.

\section{Organisation et déroulement du Som- met}

Dès lors, l'ASTED et la CLA se sont engagées à part entière dans les travaux préparatoires, en cherchant d'abord à obtenir l'appui du gouvernement canadien. Les premières demandes de subvention furent infructueuses. À l'automne 1991, grâce au soutien constant apporté par la Bibliothèque nationale, le ministère des Communications accepta de s'engager dans le projet et accorda une subvention importante pour la réalisation du Sommet. Un comité organisateur fut aussitôt mis en place, composé de Karen Adams et Ernie Ingles, respectivement directrice générale et président de la CLA, Richard Paré, président de l'ASTED, Heather Lawson, del'Association canadienne de la technologie de l'information, Bernard Dumouchel de I'ICIST, Gwynneth Evans de la Bibliothèque nationale du Canada, et Arthur Cordell, James Fulcher et Jamie Hum, du ministère des Communications. Riche de toutes les démarches, discussions, réflexions, débats et consensus antérieurs, le comité organisateur a fait preuve d'une efficacité exemplaire pour organiser en un an à peine ce Sommet dont la tenue a été fixée pour décembre 1992. Les travaux du Comité ont déjà été décrits dans d'autres publications, notamment dans l'éditorial de Richard Paré «Pour le développement d'une politique en matière d'information " paru dans Documentation et bibliothèques de janvier-mars $1993^{6}$ et dans la publication du Comité organisateur intitulé «Résumé du Sommet national sur une politique en matière d'information, 1992»7. Nous nous contenterons ici d'en rappeler les principales étapes.

Le groupe s'est d'abord préoccupé de définir clairement les objectifs du Sommet qui furent formulés en ces termes :
- élargir la compréhension et établir de nouveaux rapports et partenariats parmi les diverses composantes du secteur de l'information;

- mieux informer la population sur l'impact de l'information dans notre milieu socioéconomique canadien et surla nécessité de placer en priorité l'élaboration d'une politique en matière d'information;

- élaborer des stratégies nationales permettant d'optimiserles résultats du Sommet ${ }^{8}$.

Les étapes qui ont suivi furent les suivantes :

- préparation d'un document de réflexion qui fut transmis pour commentaires à près de cinq cents associations et institutions canadiennes et québécoises représentant les groupes de producteurs, de consommateurs, des milieux de l'information et de l'entreprise privée, des gouvernements et des bibliothèques";

- réception de quelque 150 mémoires et réponses diverses;

- compilation des réponses et rédaction, parTom Delsey de la Bibliothèque nationale du Canada, d'un document synthèse en 4 thèmes devant servir de base de discussion lors du sommet; les thèmes proposés étaient les suivants : équité de l'accès à l'information, maximisation des retombées économiques, développement des compétences individuelles et solidification de l'infrastructure;

- choix et invitation des participants au Sommet.

Le Sommet a finalement eu lieu du 6 au 8 décembre 1992 tel que prévu. II a réuni 200 participants et 100 observateurs. Les participants ont été choisis parmi les groupes suivants:

- les représentants des grands producteurs d'information: l'industrie privée, les gouvernements, les entreprises de haute technologie;

- les représentants des grands utilisateurs d'information: le secteur de l'éducation, les consommateurs, les bibliothèques et les musées tout en maintenant une re- présentation équilibrée de toutes les régions du Canada, des deux langues officielles et des minorités ${ }^{10}$.

Le Sommet a été présidé par le Dr Stuart Smith. M. André Chagnon, président du Conseil et chef de la direction du Groupe Vidéotron, a prononcé la conférence d'ouverture. Le ministre des Communications, l'Honorable Perrin Beatty, a prononcé une communication remarquée sur les télécommunications et la protection de la vie privée. Quatre conférenciers ont présenté chacun des thèmes proposés pour discussion aux participants: Marianne Scott, administratrice de la $\mathrm{Bi}$ bliothèque nationale, a traité de l'«équité d'accès»; Alan Lytle, vice-président du marketing chez Northern Telecom Canada Ltd, a abordé «la maximisation des retombées économiques»; John Godfrey, vice-président de l'Institut canadien des recherches avancées, a présenté le thème du «développement des compétences individuelles» et Georges Fierheller, président et directeur général de Rogers Cantel

5. Ont participé à cette réunion Claude Bonnelly, Université Laval; Guy Cloutier, Université de Sherbrooke; Jane Cooney, Canadian Library Association; Bernard Dumouchel, ICIST; Jane Dysart, Information Resources, Banque royale du Canada; Gwynneth Evans, Bibliothèque nationale du Canada; Sandra Houston, Macdonald Resource Centre, Conseil scolaired'Ottawa; Emie Ingles, Université de Regina; Marcel Lajeunesse, École de bibliothéconomie et des sciences de l'information, Université de Montréal; Judith MacAnanama, Hamilton Public Library; Carole Moore, Robarts Library, Université de Toronto; Pearce Penney, Newfoundland Public Library Services; Suzanne Richer, Secrétariat d'État; Philippe Sauvageau, Bibliothèque nationale $d u$ Québec; Marianne Scott, Bibliothèque nationale du Canada; Elmer Smith, ICIST; Morris Zbar, ministère des Affaires civiques et culturelles, $\mathrm{Di}$ rection des bibliothèques et de l'information.

6. Richard Paré, «Pour le développement d'une politique en matière d'information", Documentation et bibliothèques, vol. 39, no 1 (janvier-mars 1993), 3-5.

7. Résumé du Sommet national sur une politique en matière d'information, 1992, Ottawa, Canadian Library Association, 1993, 84 p.

8. Richard Paré, «Pour le développement .., 3.

9. Ibid., 4 .

10. Ibid., 4 
Mobile Communications Inc., a traité de la "solidification de l'infrastructure» ou plutôt, selon ses termes, de la «croissance de l'infratechnologie». Après chaque conférence, les participants étaient réunis en groupes restreints pour débattre du thème proposé. Des animateurs et des secrétaires de groupes étaient chargés d'encadrer les discussions, de colliger les conclusions et de préparer avec le président, le docteur Stuart Smith, la séance plénière et la synthèse finale qui fut présentée à la clôture du Sommet.

\section{Bilan, suivis et perspectives}

\section{Publication des conclusions du} Sommet : le résumé

A la suite du Sommet que nous considérons comme une réussite autant sur le plan participatif que sur le plan des échanges d'idées, le Comité organisateur s'est retrouvé devant une tâche formidable : celle de préparer, de publier et de diffuser un rapport, un résumé ou, à tout le moins, une série de recommandations découlant de cette activité. Le Comité organisateur a donc choisi de publier un résumé qui permettrait de faire ressortir le sommaire des discussions en plus des recommandations à formuler. Le défi était de taille puisque le Sommet s'était tenu à tambour battant, laissant ainsi peu de temps aux secrétaires des groupes de discussion et au président du Sommet d'étudier leurs notes et d'échanger, entre les délibérations, sur les faits saillants des discussions portant sur les quatre thèmes abordés. II y avait bien eu une séance plénière de clôture, mais seul un survol des idées principales y était ressorti. Le Comité organisateur s'est donc penché, de janvier à mars 1993, sur les notes des secrétaires afin d'en extraire les éléments principaux et d'établir un sommaire des discussions de groupe. Tout en rédigeant ces sommaires, les membres du Comité organisateur se penchaient également sur les consensus se dégageant des différents rapports des secrétaires, consensus qui seraient par la suite reformulés en recommandations. Les rédacteurs du rapport devaient avant tout s'assurer de l'objectivité de leurs propos pour refléter le plus fidèlement possible les discussions tenues lors du Sommet ainsi que les consensus qui en ont découlé. Le Résumé du Sommet national sur une politique en matière d'information, 1992 était publié en mai 1993 et diffusé à grande échelle à compter du 21 mai $^{11}$. Ce document de synthèse de 84 pages comporte essentiellement trois parties: les recommandations, le résumé des conférences, des discussions de groupe ainsi que de la séance plénière, et les annexes comprenant le document préliminaire, le sommaire des commentaires des intervenants sur la problématique et les questions à discuter lors du Sommet. Dans un communiqué de presse émis le 21 mai 1993, le Comité organisateur annonçait déjà quelques suivis et écrivait au sujet des recommandations :

Les recommandations sont regroupées sous quatre thèmes qui ont ressorti au Sommet: l'accès à l'information et la protection de la vie privée, les ressources humaines, les retombées économiques et la solidification de l'infrastructure... Les recommandations du rapport touchent un large éventail de questions se rapportant au domaine de l'information - à partir d'inclure l'équité d'accès à l'information comme un droit fondamental dans la Charte des droits et libertés, jusqu'au besoin de plus de recherche sur l'enseignement et l'étude de l'apprentissage de la pensée critique. De plus, le Sommet a appuyé le développement rapide de l'autoroute électronique au Canada, tout en demandant que les transporteurs en télécommunications réduisent leurs tarifs afin de permettre à tous les Canadiens d'utiliser cette autoroute.

En tout, quelque vingt-deux recommandations ont découlé des discussions tenues lors du Sommet. La majorité d'entre elles s'adressent aux gouvernements, fédéral autant que provinciaux, bien que plusieurs visent l'ensemble des intervenants du milieu de l'information en vue de créer ou de solidifier les liens qui devraient les unir. On trouvera en annexe le texte intégral des recommandations. En fait, ces recommandations ne forment pas en elles-mêmes ce que devrait être une politique en matière d'information au $\mathrm{Ca}$ nada. Elles définissent plutôt les premières démarches à entreprendre et à réaliser pour qu'ensuite on puisse aborder le vrai travail: celui d'élaborer une politique en matière d'information.

\section{Distribution du résumé}

La publication du résumé terminée, le Comité organisateur s'est penché sur sa distribution pour produire le plus de retombées possibles. Dans un premier temps, tous les participants, qu'ils aient été organisateurs, conférenciers, animateurs, secrétaires de groupes de discussions, délégués ou observateurs, ont reçu copie du résumé. Ce premier envoi a touché environ 250 personnes. Parla suite, le résumé a été transmis aux quelque 500 groupes approchés initialement au moment de la recherche d'un feed-back sur les thèmes à aborder lors du Sommet. On se souviendra que ces groupes représentaient des écrivains, éditeurs, bibliothécaires, fournisseurs de logiciels, de matériel informatique et de bases de données, sociétés de télécommunications, instituts de recherche et d'études politiques, consommateurs, entreprises, enseignants, conservateurs de musées et archivistes, organismes fédéraux et gouvernements provinciaux. Un envoi plus ciblé a également été entrepris. II s'adresse aux groupes ou organismes qui sont visés de près ou de loin par les recommandations. Ainsi, tous les premiers ministres, les associations de municipalités, le Conseil du trésor (fédéral), les chefs des agences dont les recommandations font mention, telles que Statistique Canada, certains comités de la Chambre des communes, ainsi que le Contrôleur général du Canada, ont fait l'objet de cet envoi ciblé.

Enfin, le Comité organisateur souhaite que le résumé obtienne la diffusion la plus vaste possible. Par le biais de la Canadian Library Association, on pourra donc obtenir sans frais des exemplaires du résumé. Il est souhaité que le résumé serve ainsi de déclencheur d'idées auprès des individus, des groupes, des associations ou des organismes qui voudront poursuivre le travail entrepris lors du sommet.

11. Résumé du Sommet national... 


\section{Le Forum sur une politique en matière d'information}

Lors de la séance plénière de clôture, les participants au Sommet considéraient qu'une première étape venait d'être franchie avec succès. Longuement planifié et vivement attendu, le Sommet ne devait pas être l'aboutissement d'un processus qui venait à peine d'être entrepris. Les participants souhaitaient créer une tribune pour discuter de ces questions à l'avenir et de façon continue. C'est ainsi que l'idée d'un forum sur une politique en matière d'information a été suggérée. On a même recommandé certaines approches pour en assurer le succès tout en suggérant sa composition éventuelle. Le Forum a donc droit à une mention spéciale dans le résumé du Sommet. À la lumière des désirs exprimés lors de la séance plénière, on y suggère également que les participants du Comité organisateur en constituent le noyau, auquel se grefferont d'autres membres par la suite. Les membres du Comité organisateur reconnaissent l'importance d'assurer un suivi au Sommet et d'établir la place que pourrait occuper un tel forum. II demeure qu'il est un peu tôt pour créer ce forum alors qu'on entreprend à peine la diffusion du résumé et que les groupes, associations ou organismes ne se sont pas ou peu encore penchés sur les suites qu'ils donneront au Sommet. Le Comité attend donc de connaître la réaction des différents intervenants avant d'entreprendre les démarches menant à la mise sur pied de ce forum. En outre, le fait est que le financement de ce forum ne pourra pas être assuré par les organismes et associations membres du Comité organisateur.

Le Forum aura sa place si les différents intervenants du milieu de l'information en assurent eux-mêmes le suivi et le réclament. Ainsi, les membres du Comité organisateur se tiendront à l'écoute du suivi donné un peu partout au pays, recueilleront l'information pertinente, verront à mousser l'actualisation des recommandations dans leur propre organisme et resteront en contact en vue d'intervenir au moment opportun. Le Comité organisateur participe également au forum d'échange d'idées électronique SUMINFO, mis en place sur le réseau Internet.

\section{Suivi des associations de biblio- thèques et de bibliothécaires}

On pouvait s'attendre à ce que les associations de bibliothécaires soient les premières à réagir à la tenue du Sommet puisqu'elles participaient depuis plus longtemps au processus. C'est effectivement ce qui est arrivé et, dès janvier 1993, le Comité organisateur recevait ses premières réactions au Sommet.

Enjanvier, la British Columbia Library Association, par l'entremise de son «BCLA Task Force on Information Policy" soumettait, à la présidente de la Canadian Library Association, ses vues et ses recommandations pour la poursuite du dialogue entrepris lors du Sommet. Elle recommandait notamment l'établissement d'une coalition des associations de bibliothécaires nationales, provinciales et régionales afin d'assurer une place prépondérante aux bibliothèques dans toute discussion à venir portant sur l'établissement d'une politique en matière d'information. Le droit du public à l'accès à l'information doit être garanti et étendu à l'avenir.

En février 1993, l'Atlantic Provinces Library Association émettait un communiqué sur le réseau Internet dans lequel elle recommandait un partenariat réel entre les différentes associations de bibliothécaires afin d'assurer le leadership nécessaire pour poursuivre le travail du Sommet. Encore là, on voulait que les bibliothécaires jouent un rôle de premier plan dans l'élaboration de toute politique en matière d'information. L'association suggérait également la tenue d'un autre sommet en 1995.

Les réactions officielles des deux associations promoteurs de l'événement, I'ASTED et la CLA, viendront au courant de l'année, une fois que les membres, réunis en congrès annuel, auront eu l'occasion de discuter des suites à donner au Sommet. Ces deux associations ont toutefois diffusé rapidement l'information entourant les événements du Sommet par des articles publiés dans Documentation et bibliothèques et dans Feliciter. Les deux conseils d'administration se sont également penchés sur le Sommet lors de leur réunion du printemps dernier. Les attentes face au rôle que devront prendre ces deux associations pour démontrer et assurer un leadership dans l'entreprise des suites concrètes au Sommet sont assez élevées. On verra, lors des congrès annuels, quelle priorité les membres des associations sont prêts à accorder aux suites à donner: devra-t-on y consacrer des fonds spéciaux? Devra-t-on y assigner des groupes de travail spécialisés? Pourra-t-on y consacrer les mêmes efforts que ceux fournis dans le cadre de la planification et la tenue du Sommet?

Suivi d'autres associations, organismes, ministères, groupes

Il est difficile, à ce stade, alors que le résumé du Sommet est en phase de distribution, de connaître les réactions ou les suivis des autres associations, organismes, ministères ou groupes. Cela est d'autant plus difficile qu'il n'existe aucun mécanisme officiel, ou même officieux, pour recueillir ces réactions. La CLA et I'ASTED agissent comme points de contact mais cela n'assure pas nécessairement qu'on les tiendra au courant automatiquement des efforts entrepris ailleurs.

On sait par contre que les membres du Comité organisateur représentant les groupes autres que les associations de bibliothécaires ont donné un suivi dans leur organisme ou association. L'Association canadienne de la technologie de l'information a déjà publié un article dans son bulletin de nouvelles et étudie les moyens d'assurer la continuité du partenariat industrie-gouvernement-associations de bibliothécaires, établi pour la préparation du Sommet. La Bibliothèque nationale du Canada et I'ICIST ont abordé le suivi à entreprendre avec leurs commissions consultatives respectives. Ces deux organismes se sont engagés à participer à tout processus qui assurera un suivi au Sommet. Quant à Communications Canada, le groupe des Nouveaux médias et télématique s'est vu chargé d'étudier les suites à donner par ce ministère. D'autres réactions ont été obtenues par le biais de lettres des différents organismes fédéraux demandant de participer à tout processus établi pour assurer le suivi au Sommet. On mentionne notamment qu'il est important de prendre part dès maintenant à ces démarches au risque de le faire trop tard. Enfin, quelques personnes ont écrit au 
ministre Perrin Beatty de Communications Canada ou aux associations promotrices pour faire part de leur satisfaction quant au Sommet et de leur intérêt à jouer un rôle quelconque dans les suites à venir.

\section{Attentes à plus long terme}

Une politique en matière d'information demeure une nécessité pour le pays. Le Canada se tourne résolument vers une économie reposant sur les connaissances pour maintenir sa prospérité en cette fin de siècle. L'industrie du secteur de l'information prend ainsi plus d'ampleur. De même, avec une population de plus en plus variée au plan socio-culturel, le pays doit conserver ou acquérir les outils nécessaires pour conserver et enrichir son patrimoine. Les individus requièrent des moyens appropriés pour obtenir et accroître leurs connaissances. Les administrations sont confrontées à des contraintes budgétaires importantes, qui les obligent à se tourner vers les solutions offertes par les technologies de l'information pour maintenir, en partie, une productivité accrue et les services exigés par la population.

Ces quelques éléments de la problématique actuelle et à venir illustrent bien la disparité des défis qui nous attendent. Jusqu'à présent, la réponse canadienne aux questions reliées de près ou de loin à l'information a été caractérisée par la fragmentation, le manque de cohérence et, surtout, l'absence de toute action commune des intervenants du milieu. Le Sommet national sur une politique en matière d'information a clairement démontré qu'un dialogue peut être établi entre tous les intervenants et que le désir de collaborer est bel et bien présent.

Notre souhait est que l'on concrétise ce désir de collaboration en donnant des suites au Sommet qui aboutiraient en une politique en matière d'information. Seule une volonté clairement exprimée par les membres de l'ASTED, de la CLA et des autres intervenants pour la poursuite des actions entreprises permettra ce suivi si nécessaire. II faudra également convaincre les gouvernements (fédéral, provinciaux et municipaux) qu'il est dans leur intérêt d'épauler les efforts dans ce domaine. Les événements des prochains mois nous permettront d'établir si le Sommet n'était qu'une fin en soi ou le début d'une ère nouvelle!
Annexe : Recommandations issues du Sommet et extraites du Résumé du Sommet national sur une politique en matière d'information, 1992.

\section{Recommandations}

Le Sommet national sur une politique en matière d'information s'est avéré une excellente tribune pour discuter de l'importance vitale de l'information et de l'échéancier qui permettra au Canada de maximiser les retombées dérivant des ressources de l'information. Le comité organisateur s'est basé sur les résumés des discussions des 10 groupes et de la plénière pour proposer les recommandations. Elles devraient amener les Canadiens à élaborer une stratégie et un plan d'action sur ces questions très complexes.

Nous recommandons que :

1. pour accroître l'accès à l'information et la protection de la vie privée

- L'équité d'accès à l'information soit reconnue comme un principe fondamental de la société démocratique et qu'elle soit incluse dans la Charte des droits et libertés.

- Le gouvernement du Canada assouplisse les droits d'auteur de la Couronne pour que plus d'information gouvernementale soit du domaine public.

- Le gouvernement du Canada crée un point d'accès unique aux informations gouvernementales, quelle qu'en soit la nature.

- Le gouvernement du Canada normalise ses contrats de licence de vente d'informations.

- Les gouvernements provinciaux et locaux mandatent et financent les bibliothèques pour qu'elles soient les portes d'entrée des autoroutes électroniques.

- Les bibliothèques publiques, en collaboration avec leurs bailleurs de fonds, revoient leur mission et leurs services pour trouver des nouveaux moyens d'atteindre les objectifs sociaux.

- Les lois et règlements fédéraux, provinciaux et municipaux soient modifiés pour permettre aux individus de refuser que des renseignements personnels obtenus par des organismes privés ou publics soient transmis à des tiers.

- Tous les gouvernements établissent des principes communs sur la vie privée et revoient leurs lois et leurs pratiques administratives pour s'assurer qu'elles correspondent bien à ces principes.

\section{2. pour développer les ressources humaines au Canada}

- À l'ère de l'information, les gouvernements fédéral et provinciaux renouvellént et réorientent leurs programmes de ressources humaines, particulièrement en éducation et en formation et leurs programmes de recherche et de développement.

- Les universités et les autres institutions de recherche entreprennent des études:

pour permettre une meilleure compréhension de l'apprentissage et l'enseignement de la pensée critique,

pour développer des méthodes de gestion de l'information efficaces et organiser des rencontres publiques sur le passage à la société d'information.

- Les éducateurs, l'industrie et d'autres organismes s'associent pour que la formation continue devienne une préoccupation commune.

- Le gouvernement, l'industrie et le milieu de l'éducation apprennent aux consommateurs comment avoir accès à l'information, comment se méfier des dangers possibles de livrer ou d'utiliser des renseignements personnels, et comment protéger la vie privée.

\section{3. pour maximiser les retombées économiques}

- Statistique Canada crée de nouveaux indices pour identifier les tendances économiques et sociales de la société d'information.

- Le gouvernement et l'industrie collaborent à la création et au développement de banques de données au Canada et sur le Canada. 
- Les gouvernements fédéral et provinciaux travaillent ensemble au développement de politiques intégrées qui mèneront à la création d'un secteur de l'information vigoureux et qu'ils harmonisent leurs politiques si nécessaire.

- Les gouvernements revoient leurs politiques fiscales, y compris les taxes comme la TPS, pour augmenter l'investissement de capital et consolider le secteur de l'information au Canada.

\section{4. pour solidifier l'infrastructure}

- Le gouvernement du Canada accélère le développement d'une autoroute électronique entièrement interconnectée et accessible à tous.

- Le gouvernement du Canada entreprenne rapidement la phase II de la révision de la Loi sur les droits d'auteur.

- Le gouvernement du Canada gère le développement de normes et de prati- ques pour la collecte, la conservation et le recouvrement d'informations historiques ou importantes pour la recherche, quelle qu'en soit la nature.

- À l'ère de la globalisation, les entreprises de télécommunication réduisent leurs tarifs relativement élevés pour favoriser l'accès à l'information et stimuler la création de nouveaux services.

\section{Aี ICOLAS}

\section{Un système de gestion}

de l'information

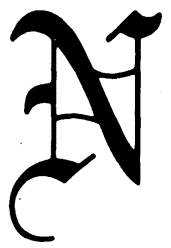

\section{Réalisé et distribué par:}

Les services informatiques

Bamyan inc.

4875, rue Rondeau

Laval, Québec

H7L 1K5

Tél.: (514) 666-0737

Fax: (514) 666-0743

\section{- Un système intégré: réseau ou monoposte}

Circulation + Catalogue + Recherche + Tri + Edition + Macro + Utilitaires de gestion; fonctionne en réseau ou en monoposte.

\section{- Un fondement pour l'avenir}

Nicolas utilise des technologies d'avant-garde et est écrit en langage C; il pourra croître et évoluer pour rencontrer vos besoins futurs.

\section{- Flexibilité}

L'usager a le loisir de bâtir son environnement selon ses propres besoins et de le modifier à volonté (définitions, rapports, etc.).

\section{- Intégrité et Efficacité}

Grâce au concept de 'longueurs variables', NICOLAS ne stocke que les données réelles réduisant ainsi l'espace-disque nécessaire. L'usage de fichier B-TREE + garantit une réponse très RAPIDE et REGULIERE aux recherches quelle que soit la grandeur des bases. Le verrouillage interne protège l'intégrité de vos données.

\section{- Installation facile}

Vous n'avez qu'à suivre le Guide d'installation. Le système est COMPLETEMENT OPERATIONNEL grâce à l'environnement complet fourni sur la disquette échantillons.

\section{- Et même plus: Les Echangeurs}

Des utilitaires de conversion puissants pour transférer les données de SDM, étudiants-Grics ou listes de la BCP. 